\title{
Methoxy-9-ellipticine lactate in refractory acute myeloid leukaemia
}

\author{
B. M. ANSARI \\ M.B., B.S., M.R.C.P., D.C.H. \\ E. N. THOMPSON \\ M.D., M.R.C.P., D.C.H. \\ Department of Child Health, The Welsh National School of Medicine, Llandough Hospital, \\ Penarth, Glamorgan
}

\begin{abstract}
Summary
Two cases of refractory acute myeloid leukaemia were treated with methoxy-9-ellipticine lactate alone and in combination with other chemotherapeutic agents. In the first patient a successful complete second and third remission was achieved lasting 2 and 3 months respectively. However, a partial remission was obtained in the second patient as treatment with methoxy-9ellipticine was only started in the terminal phase of the illness. In view of the resistance of this form of leukaemia to present chemotherapeutic drugs both initally and at the time of relapse, the response of these two cases to methoxy-9-ellipticine is worth reporting.
\end{abstract}

\section{Introduction}

Methoxy-9-ellipticine has been shown to be effective in acute myeloid leukaemia (AML) although ineffective in acute lymphatic leukaemia and Hodgkin's disease (Mathé et al., 1970). It is a potent inhibitor of DNA synthesis, but also affects RNA and protein synthesis to a lesser extent (GarciaGiralt and Macieira-Coelho, 1970). Initial remission induction is difficult in AML with at best $60 \%$ success rate using multiple drugs (Crowther et al., 1970), however, second remissions are rare. We have recently used methoxy-9-ellipticine in two children with AML and were able to induce a second remission in both. The effect in one was striking and we suggest that if this drug had been used earlier in the other patient a longer remission might have resulted. Both patients had proved refractory to other drug combinations.

The criteria for a successful complete remission were the resolution of symptoms and organomegaly with no blasts in the peripheral blood and less than $5 \%$ in the bone marrow, a neutrophil count over $1500 / \mathrm{mm}^{3}$ and the platelet count greater than $100,000 / \mathrm{mm}^{3}$. Partial remission indicated some symptomatic and haematological improvement, and failure implied no change or obvious deterioration (Ansari and Thompson, 1973).

Correspondence: Dr B. M. Ansari, Department of Child Health, The Welsh National School of Medicine, Llandough Hospital, Penarth, Glamorgan CF6 1XX, Wales.

\section{Case 1}

A 10-year-old girl developed AML in November, 1970. The initial white blood counts ranged from $19,600 / \mathrm{mm}^{3}$ to $9100 / \mathrm{mm}^{3}$ with $56 \%$ myeloblasts. Bone marrow studies showed almost complete replacement by myeloblasts which were PAS-negative and Sudan Black-positive. Remission was achieved by cyclical courses of daunorubicin, cytosine arabinoside, 6-mercaptopurine and prednisone given fortnightly for 12 weeks and then maintained on a monthly course (Table 1 ). On this regime she remained in remission for 20 months when she relapsed. An attempt to induce a second remission failed with more frequent courses of these drugs together with asparaginase. Methoxy-9-ellipticine $(40 \mathrm{mg}$ intravenously daily for 3 consecutive days each week) with prednisone (10 mg daily) was started (Fig. 1). Rapid clinical improvement resulted with confirmation of bone marrow remission after six courses of treatment, which was maintained for 2 months on daily 6-thioguanine and monthly 3-day courses of methoxy-9ellipticine. She relapsed again at the end of this period and was re-induced with prednisone $(40 \mathrm{mg} /$ $\mathrm{m}^{2} /$ day) and with courses of methoxy-9-ellipticine (40 mg), adriamycin ( $0.4 \mathrm{mg} / \mathrm{kg})$, and cyclophosphamide $\left(80 \mathrm{mg} / \mathrm{m}^{2}\right)$. These drugs were given intravenously once daily for 3 days and repeated every 10 days for six courses when a complete remission was again achieved. This third remission was maintained for 3 months with courses every 3 weeks. Further chemotherapy was subsequently unsuccessful and the patient died of refractory disease.

\section{Comment}

The treatment was well tolerated. There were no complications during induction of second remission. Transitory thrombocytopenia and neutropenia occurred at the time of the second relapse and treatment with blood and platelet infusions was required. A steady increase in neutrophil and platelet count occurred after second course of treatment with methoxy-9-ellipticine, adriamycin and cyclophosphamide which was maintained throughout the course. 

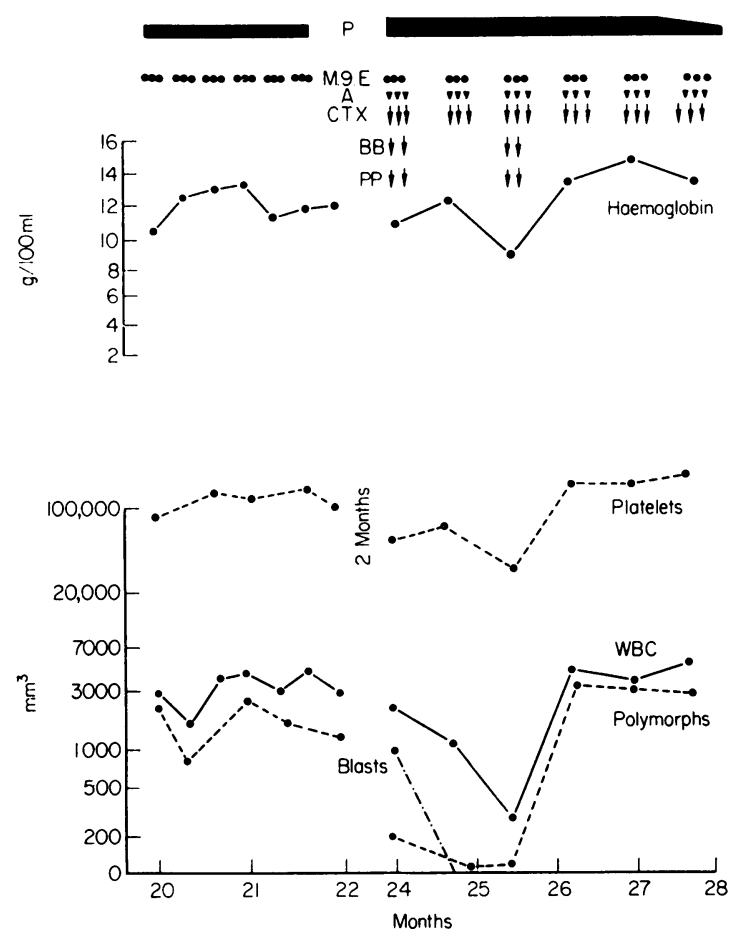

FIG. 1. Clinical progress and haematological data following treatment with methoxy-9-ellipticine. Case 1. $\mathrm{P}=$ prednisone, $\mathrm{M}-9-\mathrm{E}=$ methoxy-9-ellipticine, $\mathrm{A}=$ adriamycin, CTX = cyclophosphamide, $\mathrm{B}=$ blood transfusion, $\mathbf{P}=$ platelet transfusion.

The liver function tests, serum uric acid, blood urea and electrolytes, and ECG were normal during courses of treatment.

\section{Case 2}

A 14-year-old girl developed AML in March, 1970. Successful remission induction was achieved as with the first case and maintained monthly for 12 months (Table 1). At the time of the first relapse the disease proved resistant to more frequent courses of the initial inducing drugs and also to combination chemotherapy using cytosine arabinoside and cyclophosphamide, vincristine and prednisone, cytosine arabinoside and 6-thioguanine.

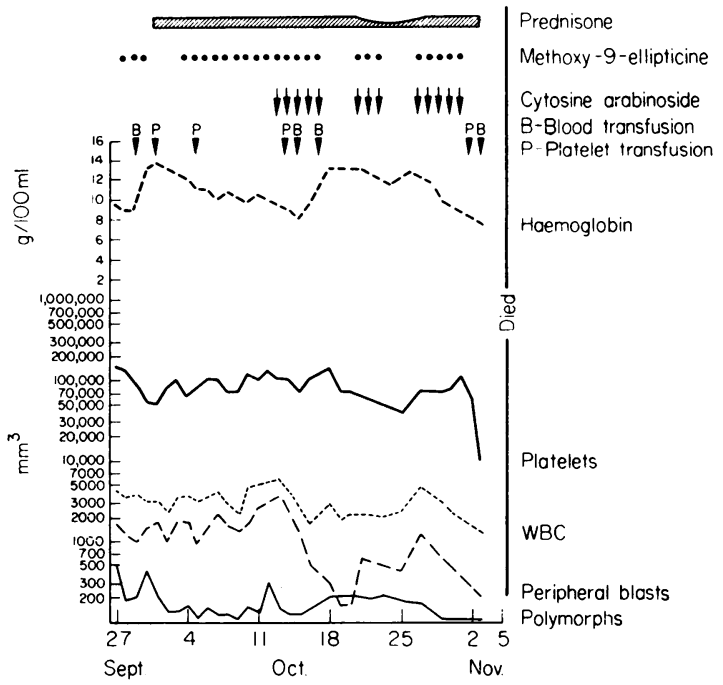

FIG. 2. Clinical progress and haematological data. Case 2.

Deterioration continued and 4 months later the bone marrow showed complete replacement with myeloblasts. Methoxy-9-ellipticine (40-80 mg intravenously once daily) and prednisone $\left(40 \mathrm{mg} / \mathrm{m}^{2} /\right.$ day) were started. Cytosine arabinoside $\left(80-130 \mathrm{mg} / \mathrm{m}^{2} /\right.$ day) was added 2 weeks later when the bone marrow showed evidence of a partial remission. The timing of drugs and the patient's clinical progress are shown in Fig. 2. The remission lasted 2 weeks only, when further deterioration occurred and she died 10 weeks after starting methoxy-9-ellipticine.

\section{Comment}

The treatment was again well tolerated. Thrombocytopenia was never very severe except towards the end; however, frequent blood and platelet transfusions were required. Hyperpyrexia reported by Mathé et al. (1970) occurred following methoxy-9ellipticine administration.

\section{Discussion}

At the time treatment was started with methoxy9-ellipticine, resistance to other drugs was well established and progression of the disease obvious.

TABLE 1. Showing drugs used initially for remission induction and maintenance chemotherapy in the two patients with acute myeloid leukaemia

\begin{tabular}{|c|c|c|c|c|}
\hline \multirow[b]{2}{*}{ Remission induction } & \multirow{2}{*}{\begin{tabular}{l}
\multicolumn{1}{c}{ Drugs } \\
Daunorubicin \\
Cytosine arabinoside \\
6-mercaptopurine \\
Prednisone
\end{tabular}} & \multirow{2}{*}{ 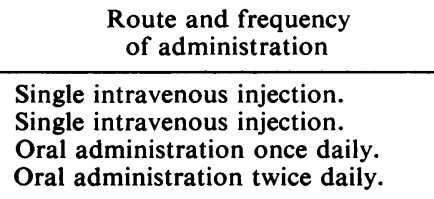 } & \multicolumn{2}{|c|}{$\begin{array}{l}\text { Dosage, timing, number and } \\
\text { frequency of courses }\end{array}$} \\
\hline & & & $\begin{array}{r}40 \mathrm{mg} / \mathrm{m}^{2} \text { Day } 1 \\
100 \mathrm{mg} / \mathrm{m}^{2} \text { Day } 1-3 \\
70 \mathrm{mg} / \mathrm{m}^{2} \text { Day } 1-5 \\
40 \mathrm{mg} / \mathrm{m}^{2} \text { Day } 1-7\end{array}$ & $\begin{array}{l}\text { Six } \\
\text { bi- } \\
\text { weekly } \\
\text { courses. }\end{array}$ \\
\hline Maintenance of remission & & As above, but drugs given monthly. & & \\
\hline
\end{tabular}


In both instances, clinical and haematological improvement was noted which was maintained in one of them. We thought that if this treatment had been started earlier in the second case the improvement may well have been sustained. However, in this instance a partial remission was achieved which was probably contributed by cytosine arabinoside. The synergistic effect of methoxy-9-ellipticine with other chemotherapeutic agents requires further evaluation. In view of the resistance of this form of leukaemia to present chemotherapeutic drugs both initially and at the time of relapse, we felt that the response of these patients to methoxy-9-ellipticine was worth reporting. However, any long-term effect cannot be excluded and in view of the poor prognosis of AML we feel a further trial of this drug is required.

\section{Acknowledgment}

The authors are extremely grateful to Professor Georges Mathé, Professor of the Faculty of Medicine of the University of Paris, for his advice and for sending methoxy-9-ellipticine for use in these cases.

\section{References}

ANSARI, B.M. \& ThOMPSON, E.N. (1973) Refractory leukemia treated with cytosine arabinoside and cyclophosphamide. Cancer, 32, 294.

Crowther, D., Bateman, C.J.T., Vartan, C.P., Whitehouse, J.M.A., Malpas, J.S., Hamilton-Fairley, G. \& Bodley SCoTt, SIR R. (1970) Combination chemotherapy using L-asparaginase, daunorubicin and cytosine arabinoside in adults with acute myelogenous leukaemia. British Medical Journal, iv, 513.

Garcia-Giralt, E. \& Macieira-Coelho, A. (1970) Methoxy9-ellipticine. II. Analysis in vitro of the mechanism of action. Revue Européenne d'Etudes cliniques et biologiques, 15, 539.

Mathé, G., Hayat, M., De Vassal, F., Schwarzenberg, L., SChneider, M., Schlumberger, J.R., JASmin, C. \& Rosenfeld, C. (1970) Methoxy-9-ellipticine lactate. III. Clinical screening: its action in acute myeloblastic leukaemia. Revue Européenne d'Etudes cliniques et biologiques, 15, 541.

\title{
Intravenous trimethoprim/sulphadimidine in the treatment of Bacteroides septicaemia
}

\author{
Gillian C. Hanson
}

\section{R. L. Woods}

\author{
Whipps Cross Hospital
}

\section{Summary}

The name Bacteroides is used to describe a group of Gram-negative bacilli which are non-sporing obligate anaerobes (Wilson and Miles, 1964). Their natural habitat is the large intestine, mouth, and vagina.

Bacteroides septicaemia is becoming increasingly well recognized as a complication of gastrointestinal and gynaecological surgery. Patients with underlying malignancy or prior antibiotic therapy are predisposed to this condition (Bodner, Koenig and Goodman, 1970). In a survey by Okubadejo, Green and Payne (1973), twenty-nine strains of $B$. fragilis were tested against co-trimoxazole - all were found to be sensitive. One of the disadvantages of co-trimoxazole has been the absence of a parenteral form for use in seriously ill patients in whom oral administration is not practicable. We report the use of intravenous trimethoprim/sulphadimidine in two cases of septicaemia due to Bacteroides infection.

Correspondence: Dr G. C. Hanson, Intensive Therapy Unit, Whipps Cross Hospital, London E11.

\section{Case reports}

\section{Case 1}

A 74-year-old man was admitted with a history of acute abdominal pain and signs suggestive of intestinal obstruction. At laparatomy, a moderately well differentiated annular carcinoma of the splenic flexure and an empyema of the gall bladder were found. Left hemicolectomy, caecostomy and a difficult cholecystectomy were performed. The patient's progress was uneventful until the seventh day postoperatively, when he became clinically shocked, with tachycardia, tachypnoea and oliguria. Blood cultures were taken and treatment with intravenous chloramphenicol commenced. Twenty-four hours later the patient's condition had further deteriorated. Treatment was changed to trimethoprim $100 \mathrm{mg}$ and sulphadimidine $500 \mathrm{mg}$ i.v. 8-hourly and i.v. cloxacillin $1.0 \mathrm{~g}$ 4-hourly. On this regime the patient steadily recovered; all subsequent blood cultures were negative. The first blood culture grew a Staphylococcus aureus of doubtful significance which on 